\title{
Stochastic Analysis and Regeneration of Rough Surfaces
}

\author{
G. R. Jafari ${ }^{a}$, S. M. Fazeli ${ }^{a}$, F. Ghasemi ${ }^{a}$, S. M. Vaez Allaei ${ }^{b}$, \\ M. Reza Rahimi Tabar ${ }^{a, c, d}$, A. Iraji zad ${ }^{a}$ and G. Kavei ${ }^{e}$ \\ ${ }^{a}$ Department of Physics, Sharif University of Technology, P.O. Box 11365-9161, Tehran, Iran \\ ${ }^{b}$ Institute for Advanced Studies in Basic Sciences, P. O. Box 45195-159, Zanjan Iran \\ c CNRS UMR 6529, Observatoire de la Côte d'Azur, BP 4229, 06304 Nice Cedex 4, France \\ ${ }^{d}$ Dept. of Physics, Iran University of Science and Technology, Narmak, Tehran 16844, Iran \\ ${ }^{-}$Material and Energy, Research Center, P. O. Box 14155-47y7, Tehran, Iran
}

\begin{abstract}
We investigate Markov property of rough surfaces. Using stochastic analysis we characterize the complexity of the surface roughness by means of a Fokker-Planck or Langevin equation. The obtained Langevin equation enables us to regenerate surfaces with similar statistical properties compared with the observed morphology by atomic force microscopy.
\end{abstract}

Studying the growth, formation and morphology of interfaces has been one of the recent interesting fields of study because of its high technical and rich theoretical advantages [1]. One of the main problems in this area is the scaling behaviour of the moments of height difference $\Delta h=h\left(x_{1}\right)-h\left(x_{2}\right)$ and the evolution of the probability density function (PDF) of $\Delta h$, i.e. $P(\Delta h, \Delta x)$ in terms of the length scale $\Delta x$. Recently Friedrich and Peinke have been able to obtain a Fokker-Planck equation describing the evolution of the probability distribution function in terms of the length scale, by analyzing some stochastic phenomena, such as turbulent free jet, etc. [2-4]. They noticed that the conditional probability density of field increments (velocity field, etc.) satisfies the ChapmanKolmogorov equation. Mathematically this is a necessary condition for the fluctuating data to be a Markovian process in the length scales [5].

In this letter using the method proposed by Friedrich and Peinke, we measure the Kramers-Moyal's (KM) coefficients for the fluctuating fields $\Delta h$ and $h(x)$ of a deposited copper film. It is shown that the first and second KM's coefficients have well-defined values, while the third and fourth order coefficients tend to zero. Therefore, by addressing the implications dictated by the theorem [5] a Fokker-Planck evolution operator has been found. The Fokker-Planck equation for $P(\Delta h, \Delta x)$ is used to give information on changing the shape of PDF as a function of the length scale $\Delta x$. By using this strategy the information of the observed intermittency of the height fluctuation is verified [6]. The first and second KM's coefficients for the fluctuations of $h(x)$, enables us to write a Langevin equation for the evolution of height with respect to $x$. Using this equation we regenerate the surface with similar statistical properties, compared with the observed morphology by atomic force microscopy. The regeneration of a surface is known as the inverse method. There are other inverse method approaches introduced in the literature [13]. In the previous attempts, to regenerate the surface, an evolution equation for $h(x, t)$ vs $t$ has been evaluated. Here we do this by an evolution equation for $h(x)$ vs $x$, for a certain time.

For this purpose, a copper film was deposited on a polished $\mathrm{Si}(100)$ substrate by the resistive evaporation method in a high vacuum chamber. The pressure during evaporation was $10^{-6}$ Torr. The thickness of the growing films was measured in situ by a quartz crystal thickness monitor. We performed all depositions at room temperature, with a deposition rate about $20-30 \mathrm{~nm} / \mathrm{min}$. The substrate temperature was determined using a chromel/alumel thermocouple mounted in close proximity of samples. The surface topography of the films was investigated using Park Scientific Instruments model Autoprobe CP. The images were collected in a constant force mode and digitized into $256 \times 256$ pixels with scanning frequency of $0.6 \mathrm{~Hz}$. The cantilever of $0.05 \mathrm{~N} \mathrm{~m}^{-1}$ spring constant with a commercial standard pyramidal $S i_{3} N_{4}$ tips was used. A variety of scans, each with size $L$ were recorded at random locations on the $\mathrm{Cu}$ film surface.

It is a common procedure to characterize the complexity of a rough surface by checking the scaling behaviour of the moments $C_{q}=<\left|h\left(x_{1}\right)-h\left(x_{2}\right)\right|^{q}>$ in terms of the length scale $\Delta x=\left|x_{1}-x_{2}\right|$. We investigated the scaling behaviour of the q-th moment $C_{q}$ and observed that all of the moments (up to $q=20$ ) behave as $\left|x_{1}-x_{2}\right|^{\xi_{q}}$ within the scaling region $\sim 10$ to $150 \mathrm{~nm}$. We have found a nonlinear relation between $\xi_{q}$ and $q$. This shows that the height fluctuations are intermittent or multi-fractal ( see $[8,15]$ and references therein). The roughness exponent $\alpha$ is related to the exponent $\xi_{2}$ as $\alpha=\xi_{2} / 2$ [1]. For the stationary samples with thickness $440 \mathrm{~nm}$, the roughness exponent $\alpha$ was found to be $0.83 \pm 0.03$. From the stochastic point of view one has to remark that multi-fractality is based on properties of the roughness on distinct length scales. However, checking the scaling behaviour does not explain possible correlation between the 


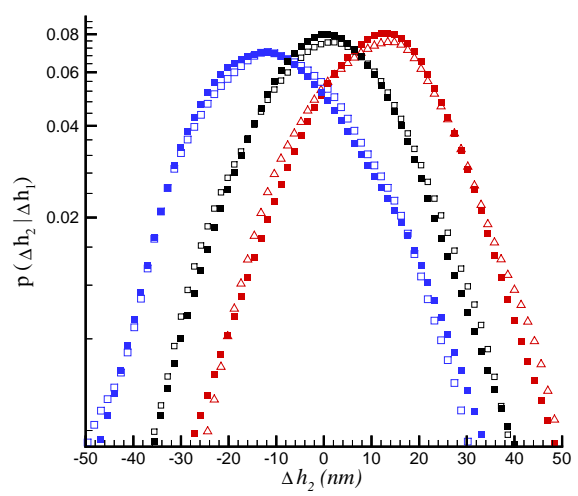

FIG. 1. Test of Chapman-Kolmogorov equation for different values $\Delta h_{1}=-21 \mathrm{~nm}, \Delta h_{1}=0$ and $\Delta h_{1}=21 \mathrm{~nm}$. The bold and open symbols represent directly evaluated PDF and the integrated PDF, respectively. The length scales $\Delta x_{1}, \Delta x_{2}$ and $\Delta x_{3}$ are $180 \mathrm{~nm}, 320 \mathrm{~nm}$ and $260 \mathrm{~nm}$, respectively.

roughness measures on different scales. Also it is noted that the methods based on multi-fractality are limited to the subclass of rough surfaces which show scaling properties. The method introduced by Friedrich and Peinke is a general method, which explains the complexity of the surface roughness, with no scaling feature to be explicitly required. Their method yields an estimation of an effective stochastic equation in the form of a FokkerPlanck equation (also known as Kolmogorov equation). The connection between the multifractality and Markovianity has been discussed in [4].

A complete characterization of the statistical properties of the height fluctuation requires the evaluation of joint $\mathrm{PDF}^{\text {'s }} P_{N}\left(\Delta h_{1}, \Delta x_{1} ; \ldots ; \Delta h_{N}, \Delta x_{N}\right)$, for any arbitrarily $N$. If the process is a Markov process (a process without memory), an important simplification arises. For this type process the N-point joint PDF, $P_{N}$, is generated by a product of the conditional probabilities $P\left(\Delta h_{i+1}, \Delta x_{i+1} \mid \Delta h_{i}, \Delta x_{i}\right)$, for $i=1, \ldots, N-1$. As a necessary condition for being a Markov process, the Chapman-Kolmogorov equation,

$$
\begin{aligned}
& p\left(\Delta h_{2}, \Delta x_{2} \mid \Delta h_{1}, \Delta x_{1}\right)= \\
& \int \mathrm{d}\left(\Delta h_{3}\right) p\left(\Delta h_{2}, \Delta x_{2} \mid \Delta h_{3}, \Delta x_{3}\right) p\left(\Delta h_{3}, \Delta x_{3} \mid \Delta h_{1}, \Delta x_{1}\right)
\end{aligned}
$$

should hold for any value of $\Delta x_{3}$, in the interval $\Delta x_{2}<\Delta x_{3}<\Delta x_{1}[5]$. We checked the validity of the Chapman-Kolmogorov equation for different $\Delta h_{1}$ triplets by comparing the directly evaluated conditional probability distributions $p\left(\Delta h_{2}, \Delta x_{2} \mid \Delta h_{1}, \Delta x_{1}\right)$ with the ones calculated according to rhs. of eq.(1). In Fig. (1), the two direct and integrated PDF's are superimposed for the purpose of illustration. The bold and open symbols represent directly evaluated PDF and the integrated PDF, respectively. Assuming a statistical error of the square
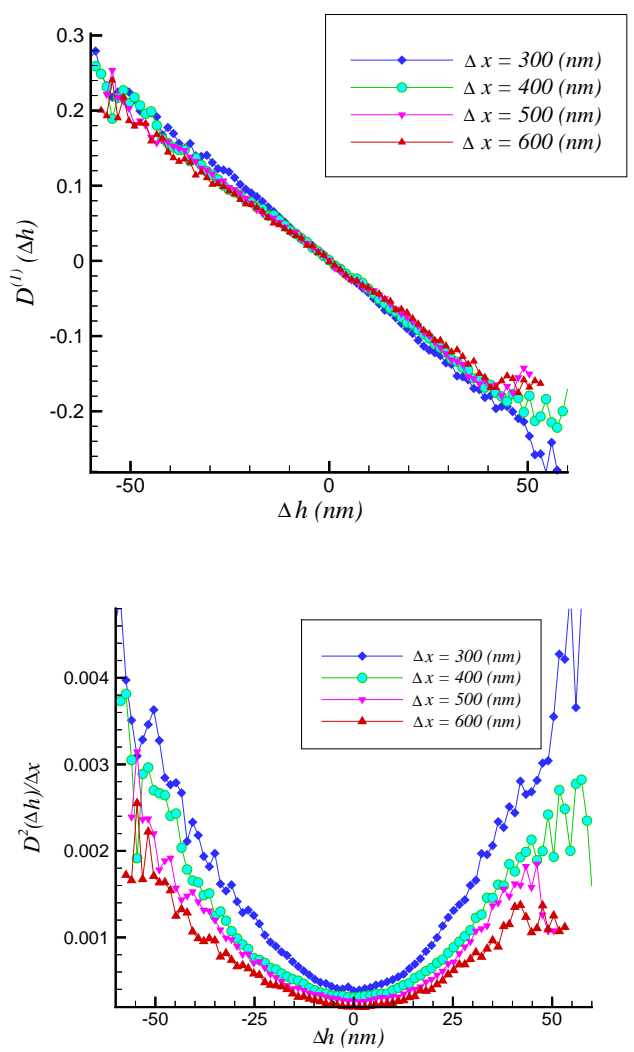

FIG. 2. Drift and diffusion coefficients $D^{1}(\Delta h)$ and $D^{2}(\Delta h)$ are estimated from the eq. (3). The $D^{1}$ and $D^{2}$ present the linear and quadratic behavior, respectively.

root of the number of events of each bin we find that both PDF's are statistically identical (see also [14] an another interesting and carefully presented example of application of the Chapman-Kolmogorov equation).

It is well-known, the Chapman-Kolmogorov equation yields an evolution equation for the change of the distribution function $p(\Delta h, \Delta x)$ across the scales $\Delta x$. The Chapman-Kolmogorov equation formulated in differential form yields a master equation, which can take the form of a Fokker-Planck equation [5]:

$$
\begin{aligned}
& \frac{d}{d r} p(\Delta h, r)= \\
& {\left[-\frac{\partial}{\partial \Delta h} D^{(1)}(\Delta h, r)+\frac{\partial^{2}}{\partial \Delta h^{2}} D^{(2)}(\Delta h, r)\right] p(\Delta h, r)}
\end{aligned}
$$

where $r:=\Delta x$. The drift and diffusion coefficients $D^{(1)}(\Delta h, r), D^{(2)}(\Delta h, r)$ can be estimated directly from the data and the moments $M^{(k)}$ of the conditional probability distributions:

$$
\begin{aligned}
& D^{(k)}(\Delta h, r)=\frac{1}{k !} \lim _{\Delta r \rightarrow 0} M^{(k)} \\
& M^{(k)}=\frac{1}{\Delta r} \int d \Delta h^{\prime}\left(\Delta h^{\prime}-\Delta h\right)^{k} p\left(\Delta h^{\prime}, r+\Delta r \mid \Delta h, r\right)
\end{aligned}
$$




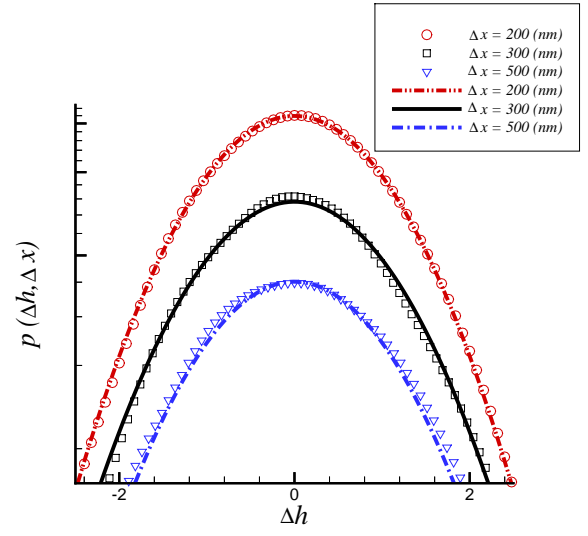

FIG. 3. Probability densities of the height difference $\Delta h=h(x+\Delta x)-h(x)$ for the length scales $\Delta x=200,300$ and $500 \mathrm{~nm}$ (from top to bottom). The results obtains from the data analysis of the AFM image (R) and numerical integration of an effective Fokker-Planck equation (E) i.e. eq.(2), respectively. The $\mathrm{PDF}^{\prime} \mathrm{s}$ are shifted in vertical directions for convenience of presentation and $\Delta h^{6}$ s are measured in units of the standard deviation of $\Delta h$ at $\Delta x=200 \mathrm{~nm}$.

The coefficients $D^{(k)}(\Delta h, r)^{\text {ss }}$ are known as KramersMoyal coefficients. The drift and diffusion coefficients $D^{(1)}$ and $D^{(2)}$ are displayed in fig.(2). It turns out that the drift term $D^{(1)}$ is a linear function of $\Delta h$, whereas the diffusion term $D^{(2)}$ is a function quadratic in $\Delta h$. For large values of $\Delta h$, our estimation becomes poor and thus uncertainty increases. From the analysis of the data set we obtain the following approximation:

$D^{(1)}(\Delta h, \Delta x)=-0.0055 \Delta h$

$D^{(2)}(\Delta h, \Delta x)=\left[\left(2.9 \times 10^{-4}\right)(\Delta h)^{2}+0.015(\Delta x)^{0.45}\right] / \Delta x$

where $\Delta h$ is measured in units of the standard deviation of $\Delta h$ at $\Delta x=200 \mathrm{~nm}$. According to Pawula's theorem, the Kramers-Moyal expansion stops after the second term, provided that the fourth order coefficient $D^{(4)}(\Delta h, \Delta x)$ vanishes [5]. The forth order coefficients $D^{(4)}$ in our analysis was found to be about $D^{(4)} \simeq$ $10^{-4} D^{(2)}$. In this approximation we can ignore the coefficients $D^{(n)}$ for $n \geq 3$. To perform a quantitative test of the result with these coefficients, we solve the FokkerPlanck equation for the PDF at scales $\Delta x \ll L$ with a given distribution at sample size $L[6,7]$. Fig.(3) shows a comparison between the analysis of AFM image and the solutions of the obtained Fokker-Planck equation for the copper surface for the length scales $\Delta x=200,300$ and $500 \mathrm{~nm}$. The figure shows that the solutions of our model fit the experimentally determined PDF's with good precision. In the integral scale our measured PDF is nearly a Gaussian distribution. In our approximation the stochastic process underlying the height fluctuation changes is a linear stochastic process with multiplicative noise.

By the same procedure, we checked the Markovian nature of the fluctuations of the height $h=h(x)-\bar{h}$, and found the following expression for the $D^{(1)}(h)$ and $D^{(2)}(h)$ :

$$
\begin{aligned}
& D^{(1)}(h)=-0.01 h \\
& D^{(2)}(h)=0.088-0.004 h+5.19 \times 10^{-5} h^{2} .
\end{aligned}
$$

The height field is measured in units of the standard deviation of $h$. Analogous to equation (2), we can write a Fokker-Planck equation for the PDF of $h$ by replacing $r$ and $\Delta h$ with $x$ and $h$, respectively. We note that this Fokker-Planck equation is equivalent to the following Langevin equation (using the Ito interpretation) [5]:

$$
\frac{d}{d x} h(x)=D^{(1)}(h)+\sqrt{D^{(2)}(h)} f(x) \quad .
$$

Here, $f(x)$ is a random force, zero mean with gaussian statistics, $\delta$-correlated in $x$, i.e. $\left\langle f(x) f\left(x^{\prime}\right)\right\rangle=\delta(x-$ $\left.x^{\prime}\right)$. Furthermore, with this last expression, it becomes clear that we are able to separate the deterministic and the noisy components of the surface height fluctuations in terms of the coefficients $D^{(1)}$ and $D^{(2)}$. Equation (6) enables us to regenerate rough surfaces which are similar to the original one (in the statistical sense ). In fig. (4) the AFM and regenerated images are demonstrated. The regenerated surface is very similar in statistical sense to the original one. All regenerated patterns are statistically similar. To ensure this fact, for instance, in Fig.(5) we have plotted the second moment of the structure function $C_{2}$ for the AFM and a regenerated surfaces and their roughness exponents were found $0.83 \pm 0.03$ and $0.83 \pm$ 0.01 , respectively.

(4) There are a few comments on the regeneration of rough surface that we would like to notify. When we are discussing about a Markov process, one should note that, this is true within an approximation. For instance, in random motion of a particle inside a fluid, it is known that the collision of the particle with the fluid molecules is not instantaneous, and takes a certain duration. During the time that a collision is taking place, the change of velocity is not Markov, because the velocities in the collision time scale have memory. Consequently, in the time series for the velocities of the particle, if the time intervals are less than the collision time scale, the process can not be regarded as a Markov process. The minimum time interval that the particle motion can be considered as a Markov process is known as Markov time scale and the motion is known as a Brownian motion. In the stochastic analysis of the rough surface, we are dealing with the Markov property of height fluctuations in spatial dimensions, therefore, instead of a Markov time scale, here we will have a Markov length scale $l_{\text {markov }}$. Our analysis shows that $l_{\text {markov }} \simeq 160 \mathrm{~nm}$, equivalent to 8 pixels in our AFM image [9]. The surface is regenerated by iterating the eq.(6), which gives us a series of data without 

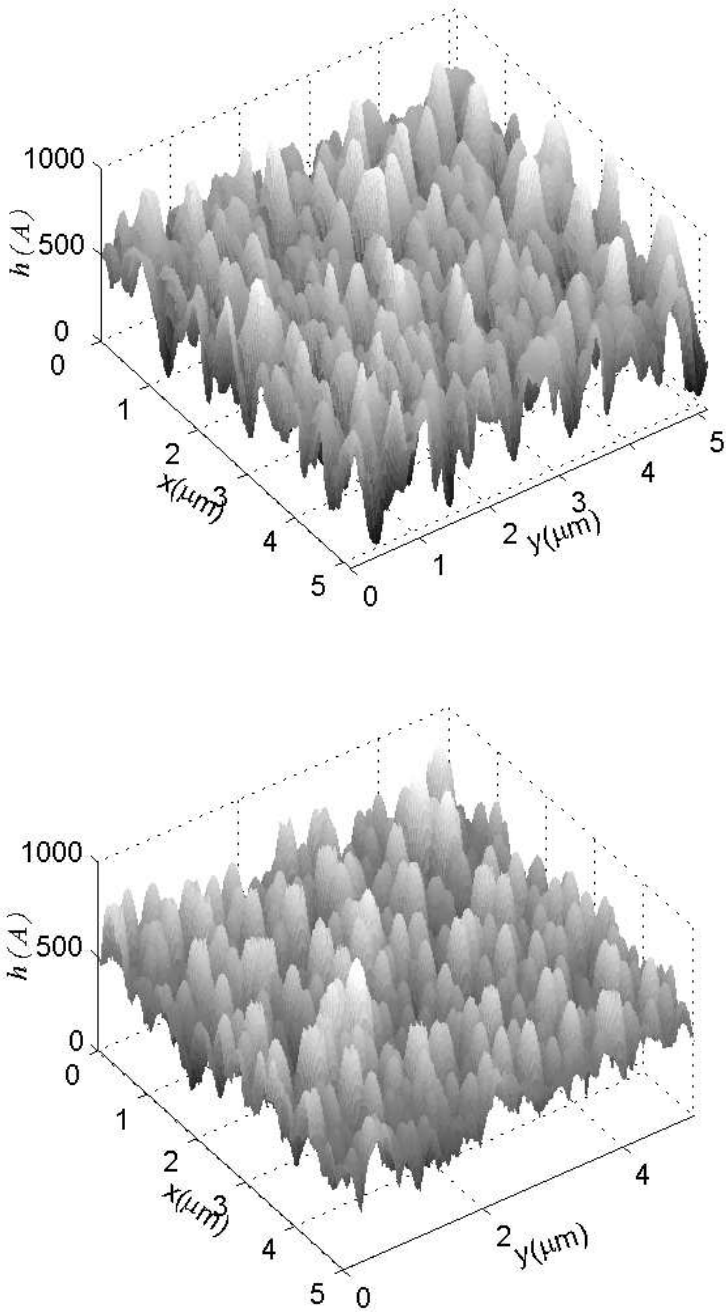

FIG. 4. AFM and regenerated surface images (from up to bottom), which we have regenerated the rough surface using the Langevin equation for dynamics of $\mathrm{h}(\mathrm{x})$. As drift term $D^{(1)}(h)=-0.01 h$ and as diffusion term $D^{(2)}(h)=0.088-0.004 h+5.19 \times 10^{-5} h^{2}$.

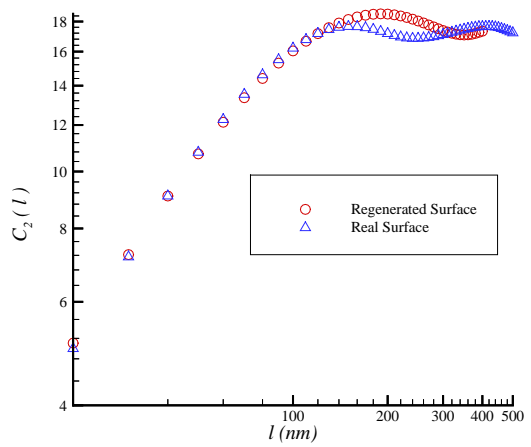

FIG. 5. Log-log plot of the second moment of height-difference vs $l$, for real sample and regenerated sample. The roughness exponents for real and regenerated are $0.83 \pm 0.03$ and $0.83 \pm 0.01$, respectively. memory. To compare the regenerated surface with the original one, we have to take the spatial interval in the numerical discritization of eq.(6), to be equal to 1 pixel. However, here the Markov length is equal to 8 pixels. Therefore, we should relate the height field within the Markov length. There are a number of methods to correlate the generated data in this interval [9]. We do this by means of scanning the surface with a tip, where its size is about the Markov length [12]. The tip that we have used has the form $z=a x^{2}+b y^{2}$. In this case the parameters $a$ and $b$ are 0.035 .

In summary, we have shown that the probability density of height increments satisfy a Fokker-Planck equation which encodes the Markovian property of these fluctuations in a necessary way. We are able to give the expression of Kramers-Moyal's coefficients for the stochastic processes $\Delta h$ and $h$ by using the polynomial ansatz $[10,11]$. Also we could find the form of path probability functional of the height increments in spatial scale, which naturally encodes the scale dependence of probability density. This gives a clear picture about the intermittent nature in height fluctuations. The methods enables us to regenerate many realizations of the rough surface with similar statistical properties in favored scales. As an application, large surface generation would be possible by sampling the real surface with high resolution (in same the resolution as nanoscope imaging, e.g. AFM images). This would be applicable in computer simulation of the surface and interface processes, for example, the diffusion of materials between rough surfaces, the effect of roughness on the friction, and so on.

We thank F. Azami, M. Sahimi, and N. Taghavinia for their useful discussions.

[1] J. Krug and H. Spohn in "Solids Far from Equilibrium Growth, Morphology and Defects", edited by C. Godreche (Cambridge University Press, New York, 1990); A.-L. Barabasi and H. E. Stanley, "Fractal Concepts in Surface Growth" (Cambridge University Press, New York, 1995) ; T. Halpin-Healy and Y. C. Zhang, Phys. Rep.245,218(1995).

[2] R. Friedrich and J. Peinke Phys. Rev. Lett. 78, 863 (1997)

[3] R. Friedrich, J. Peinke and C. Renner Phys. Rev. Lett. $\mathbf{8 4}(22), 5224$ (2000)

[4] R. Friedrich, K.Marzinzik and A.Schmigel, in "A perspective Look at Nonlinear Media", Edited by Jurgen Parisi, Stefan C. Muller and Walter Zimmermann, Lecture notes in Physics, Vol. 503, P. 313 (Springer-verlag, Berlin, 1997).;R. Friedrich, S. Siegert, J. Peinke, etal. Physics Letters A, 271(3):217, (2000).

[5] H. Risken "The Fokker-Planck equation" Springer, Berlin, 1984. 
[6] J. Davoudi and M. Reza Rahimi Tabar, Phys. Rev. Lett. 82, 1680 (1999)

[7] A. A. Donkov, A.D. Donkov and E.I. Grancharova, mathph/9807010, math-phys/9807011

[8] A. Iraji zad, G. Kavei, M. Reza Rahimi Tabar and S.M. Vaez Allaei, J. Phys. : Condensed Matter 151889 (2003)

[9] C. Renner, J. Peinke, and R. Friedrich. Journal of Fluid Mechanics, 433 383, (2001); cond-mat/0102494.

[10] M. Ragwitz and H. Kantz, Phys. Rev. Lett. 87, 254501 (2001)

[11] R. Friedrich, C. Renner, M.Siefert and J. Peinke, physics/0203005

[12] J. Aue and J. Th. M. De Hosson, Appl. Phys. Lett 71 (10) 1347 (1997)

[13] C-H. Lam and L.M. Sander, Phys. Rev. Lett 71, 561 (1993); A. Giacometti and M. Rossi, Phys. Pev. E 63 046102 (2001)

[14] A. Fuliski, Z. Grzywna, I. Mellor, Z. Siwy, and P. N. R. Usherwood, Phys. Rev. E., 58 919, 1998.

[15] S. Mercik and K. Weron, Phys. Rev. E 63 051910, 2001 